\title{
Growth of respiratory droplets in cold and humid air
}

\author{
Chong Shen $\mathrm{Ng} \odot,{ }^{1,2, *}$ Kai Leong Chong $\odot,{ }^{1,2, *}$ Rui Yang $\odot,{ }^{1,2}$ Mogeng Li $\odot,{ }^{1,2}$ \\ Roberto Verzicco $\left(1,{ }^{1,2,3,4}\right.$ and Detlef Lohse ${ }^{1,1,2, \dagger}$ \\ ${ }^{1}$ Physics of Fluids Group, Max Planck Center for Complex Fluid Dynamics, J. M. Burgers Center for Fluid \\ Dynamics and MESA+ Research Institute, Department of Science and Technology, University of Twente, \\ 7500 AE Enschede, The Netherlands \\ ${ }^{2}$ Max Planck Institute for Dynamics and Self-Organisation, 37077 Göttingen, Germany \\ ${ }^{3}$ Dipartimento di Ingegneria Industriale, University of Rome "Tor Vergata," Roma 00133, Italy \\ ${ }^{4}$ Gran Sasso Science Institute, Viale F. Crispi, 767100 L'Aquila, Italy
}

(Received 2 December 2020; accepted 14 April 2021; published 21 May 2021)

\begin{abstract}
The ambient conditions surrounding liquid droplets determine their growth or shrinkage. However, the precise fate of a liquid droplet expelled from a respiratory puff as dictated by its surroundings and the puff itself has not yet been fully quantified. From the view of airborne disease transmission, such as SARS-CoV-2, knowledge of such dependencies is critical. Here, we employ direct numerical simulations (DNS) of a turbulent respiratory vapor puff and account for the mass and temperature exchange with respiratory droplets and aerosols. In particular, we investigate how droplets respond to different ambient temperatures and relative humidity $(\mathrm{RH})$ by tracking their Lagrangian statistics. We reveal and quantify that in cold and humid environments, as there the respiratory puff is supersaturated, expelled droplets can first experience significant growth, and only later followed by shrinkage, in contrast to the monotonic shrinkage of droplets as expected from the classical view by Wells in 1934. Indeed, cold and humid environments diminish the ability of air to hold water vapor, thus causing the respiratory vapor puff to supersaturate. Consequently, the supersaturated vapor field drives the growth of droplets that are caught and transported within the humid puff. To analytically predict the likelihood for droplet growth, we propose a model for the axial RH based on the assumption of a quasistationary jet. Our model correctly predicts supersaturated RH conditions and is in good quantitative agreement with our DNS. Our results culminate in a temperature-RH map that can be employed as an indicator for droplet growth or shrinkage.
\end{abstract}

DOI: 10.1103/PhysRevFluids.6.054303

\section{INTRODUCTION}

The flow physics of a respiratory droplet is crucial to understand the airborne transmission of respiratory diseases [1,2], and recently due to the pandemic of COVID-19, the interest in this subject has been renewed [3-12]. Tiny saliva and mucus droplets, mostly of the size of tens to hundreds of micrometers $[13,14]$, are expelled from the mouth at speaking, screaming, shouting, singing,

\footnotetext{
*These authors contributed equally to this work.
}

†.lohse@utwente.nl

Published by the American Physical Society under the terms of the Creative Commons Attribution 4.0 International license. Further distribution of this work must maintain attribution to the author(s) and the published article's title, journal citation, and DOI. Open access publication funded by the Max Planck Society. 
coughing, sneezing, or even breathing. The expelled microsized droplets have long been recognized to be the carriers for viruses which are responsible for the viral transmission from one host to others [15].

Airborne disease transmission is a difficult subject because many intricate factors simultaneously affect the transmission and a complete understanding requires a multidisciplinary collaboration [16,17]. In addition to the understanding from epidemiology [18-20] and virology [21], fluid mechanics also offers essential insight into the mechanisms of airborne disease transmission [2]. However, surprisingly little is known on the transport and fate of the respiratory droplets, once they have been expelled from the mouth. For example, how the air temperature and the relative humidity influences the actual fate of the droplets remains an open question. It is a complicated process involving interactions between turbulent eddies, a vapor field, and a temperature field with the respiratory droplets. Therefore, accurate quantification of these effects is necessary.

On the fate of respiratory droplets in fluid flow, classical work was done by Wells in the 1930s $[22,23]$. The picture that Wells developed, at that time in connection with the transmission of tuberculosis, considers only the settling and evaporation of the droplets. After droplets are expelled from the mouth, they settle to the ground by gravity, and, in this simple model, they evaporate with their surface area decreasing linearly with time, the so-called $d^{2}$ law [24]. Then for a given ambient temperature and relative humidity, one can estimate the lifetime of the respiratory droplet based on the $d^{2}$ law.

However, in an actual respiratory event, the assumptions of settling and evaporation are insufficient to describe the full physical process. In reality, more complicated flows are possible: Abkarian et al. [25] demonstrated that speech can produce jetlike flow transport, and Bhagat et al. [11] illustrated that body or breathing plumes from a person can also spread droplet nuclei in enclosed spaces. Recently, a new paradigm has been suggested by Bourouiba et al. $[1,3,26]$, which states that instead of the isolated droplet being considered, one should also take into account the importance of turbulent vapor puffs. Inspired by this paradigm, using direct numerical simulations, Chong et al. [27] showed that droplets have $O(100)$ longer lifetimes in a turbulent vapor puff at high ambient relative humidity than that predicted by the $d^{2}$ law. Past numerical studies that consider different ambient conditions typically adopt mean flow modeling approaches [28-30]. The role of ambient conditions requires large-scale parametrizations [31-33], but run the risk of obscuring the underlying physics. Newer higher-fidelity direct numerical simulations (DNS) with fully coupled turbulence are emerging [34-36], but full quantification of the Lagrangian statistics is still limited.

In this paper, we aim at quantifying the effect of ambient temperature $\left(\theta_{\mathrm{amb}}\right)$ and relative humidity $\left(\mathrm{RH}_{\mathrm{amb}}\right)$ on respiratory droplets using direct numerical simulations of a turbulent puff. Normal coughing with an actual injection profile has been simulated with 5000 droplets being tracked by the Euler-Lagrangian approach. We reveal a regime in which the respiratory droplets considerably grow before their shrinkage due to evaporation. The droplet growth happens under specific combinations of ambient conditions, preferentially in a cold and humid environment. To predict the criteria of having droplet growth, we further propose a theoretical model to estimate the local relative humidity experienced by the droplets, which agrees excellently with our simulation results. While our model can help to formulate public health guidelines or indoor ventilation strategies in order to mitigate the spreading of respiratory droplets, the infectivity of virus-containing droplets and droplet nuclei, which remain after evaporation, still requires further investigation, in particular on the virus dose required for infections and whether dried-out droplet nuclei may also be infectious. This issue remains controversial [15] and cannot be answered within a fluid dynamical study.

The paper is organized as follows: In Sec. II we give the governing equations, followed by the simulations details in Sec. III. Our main numerical results- the effect of ambient temperature on a turbulent vapor puff and on the droplets-are given in Sec. IV. Section V supplies the Lagrangian statistics of the droplets, including the relative humidity around them. This relative humidity around the droplet can also be derived within a theoretical model, which is derived in Sec. VI and then compared with the numerical data and applied to predict the local relative humidity as a function 
of the control parameter ambient temperature and ambient relative humidity. The paper ends with conclusions (Sec. VII).

\section{GOVERNING EQUATIONS}

We consider an incompressible flow $\left(\partial u_{i} / \partial x_{i}=0\right)$ of a gas phase, with both temperature and vapor concentrations coupled to the velocity field by employing the Boussinesq approximation. The governing equations read

$$
\begin{gathered}
\frac{\partial u_{i}}{\partial t}+u_{j} \frac{\partial u_{i}}{\partial x_{j}}=-\frac{\partial p}{\partial x_{i}}+v_{\text {air }} \frac{\partial^{2} u_{i}}{\partial x_{j}^{2}}+g\left(\beta_{\theta} \theta+\beta_{c} c\right) \hat{e}_{y} \\
\rho_{g} c_{p, g}\left(\frac{\partial \theta_{g}}{\partial t}+u_{i} \frac{\partial \theta_{g}}{\partial x_{i}}\right)=k_{g} \frac{\partial^{2} \theta_{g}}{\partial x_{i}^{2}}-\sum_{n=1}^{N} c_{p, g} \theta_{g, n} \frac{d m_{n}}{d t} \delta\left(\vec{x}-\vec{x}_{n}\right)-\sum_{n=1}^{N} h_{m} A_{n}\left(\theta_{g, n}-\theta_{n}\right) \delta\left(\vec{x}-\vec{x}_{n}\right) \\
\frac{\partial c}{\partial t}+u_{i} \frac{\partial c}{\partial x_{i}}=D_{\text {vap }} \frac{\partial^{2} c}{\partial x_{i}^{2}}-\sum_{n=1}^{N}\left(\frac{\rho_{l}}{\rho_{g}} A_{n} \frac{d r_{n}}{d t} \delta\left(\vec{x}-\vec{x}_{n}\right)\right)
\end{gathered}
$$

The gas phase is solved using DNS by a staggered second-order accurate finite-difference scheme and marched in time using a fractional-step third-order Runge-Kutta approach [37,38]. For droplets, we apply the spherical point-particle model, and consider the conservation of momentum (MaxeyRiley equation [39]), energy, and mass as follows:

$$
\begin{gathered}
\frac{d u_{i, n}}{d t}=(\beta+1) \frac{D u_{i, g, n}}{D t}+(\beta+1) \frac{3 v_{\text {air }}\left(u_{i, g, n}-u_{i, n}\right)}{r_{n}^{2}} f_{d}+g \beta \hat{e}_{y}, \\
\rho_{l} c_{p, l} V_{n} \frac{d \theta_{n}}{d t}=\rho_{l} A_{n} L \frac{d r_{n}}{d t}+h_{m} A_{n}\left(\theta_{g, n}-\theta_{n}\right), \\
\frac{d r_{n}}{d t}=-\frac{D_{\text {vap }} S h_{\text {drop }}}{2 r_{n}} \frac{\rho_{g}}{\rho_{l}} \ln \left(\frac{1-c_{\text {fluid }}}{1-c_{\text {drop }}}\right) .
\end{gathered}
$$

The time integration of Eqs. (4)-(6) is done by a second-order Adams-Bashforth scheme. The droplets are coupled to the vapor and temperature field by the source terms in Eqs. (2) and (3) (see the last terms on the right-hand side). The notations we used in equations are as follows: $u_{i}, u_{i, n}$, and $u_{i, g, n}$ are the velocities of gas, droplets, and gas at the location of the droplets, respectively. $\theta_{g}, \theta_{n}$, and $\theta_{g, n}$ are in degrees Kelvin and used to represent the temperature of gas, droplets, and gas at the location of the droplets, respectively. As the thermal diffusive timescale for the microsized water droplet is $\left(10 \times 10^{-6}\right)^{2} \mathrm{~m}^{2} / 10^{-7} \mathrm{~m}^{2} \mathrm{~s}^{-1} \sim O(\mathrm{~ms})$, it is reasonable to assume that the temperature inside the droplets is uniform for this problem. $c$ is the vapor mass fraction, $r_{n}$ the droplet radius, $A_{n}$ the surface area of the droplets, $V_{n}$ the volume of the droplets, and $m_{n}$ the mass of the droplets. Also, $p$ denotes the reduced pressure. $h_{m}$ is the heat transfer coefficient. $L$ is the latent heat of vaporization of the liquid. $\rho_{l}$ and $c_{p, l}$ are the density and specific heat capacity of the droplets assumed to consist of water. $v_{\text {air }}$ is the kinematic viscosity of air. $k_{g}$ is the thermal conductivity of gas which relates to the thermal diffusivity of gas $D_{g}$ by $k_{g} \equiv D_{g} \rho_{g} c_{p, g}$. $\rho_{g}$ and $c_{p, g}$ are the density and specific heat capacity of gas. Note that $\rho_{g} c_{p, g}=\left(\rho_{a} c_{p, a}+\rho_{v} c_{p, v}\right)$, with $\rho_{a}$ and $\rho_{v}$ being the densities of air and vapor and $c_{p, a}$ and $c_{p, v}$ being the specific heat capacities of air and vapor. $c_{\text {drop }}$ and $c_{\text {fluid }}$ denote the vapor mass fractions of the droplet and the fluid at the location of the droplet. $\beta$ is a dimensionless measure of the droplet density relative to the fluid density, and is defined as $\beta \equiv 3 \rho_{g} /\left(\rho_{g}+2 \rho_{l}\right)-1 . f_{d}$ is the prefactor for the drag corrections defined as $f_{d}=1+0.169 \mathrm{Re}_{\mathrm{drop}}^{2 / 3}[40]$. 
The estimations of $h_{m}$ and $S h_{\text {drop }}$ for a single spherical droplet are given by the Ranz-Marshall correlations [41],

$$
\begin{gathered}
S h_{\text {drop }}=2+0.6 \operatorname{Re}_{\text {drop }}^{1 / 2}\left(\nu_{\text {air }} / D_{\text {vap }}\right)^{1 / 3}, \\
h_{m} r /\left(D_{g} c_{p, g} \rho_{g}\right)=2+0.6 \operatorname{Re}_{\text {drop }}^{1 / 2}\left(v_{\text {air }} / D_{g}\right)^{1 / 3},
\end{gathered}
$$

where we have a droplet Reynolds number, $\operatorname{Re}_{\text {drop }}=\left|u_{i, g, n}-u_{i, n}\right|(2 r) / \nu_{\text {air }}$.

To calculate the local relative humidity, the saturated vapor mass fraction $c_{\text {sat,vap }}$ is determined by the ideal gas law $c_{\text {sat,vap }}=P_{\text {sat }} /\left(\rho_{g} R \theta_{g}\right)$, where $R$ is the specific gas constant of water vapor and $P_{\text {sat }}$ is the saturated vapor pressure, determined through Antoine's relation as $P_{\text {sat }}\left(\theta_{g}\right)=10^{5} \exp \left[11.6834-3816.44 /\left(226.87+\theta_{g} / \mathrm{K}-273.15\right)\right]$. In principle, the ClausiusClapeyron formula could also be used in our simulations. The Antoine equation is derived from the Clausius-Clapeyron formula and applies a small correction in order to obtain a more reliable result for larger temperature intervals.

\section{SIMULATION DETAILS}

The computational domain size is $0.37 \mathrm{~m}$ (spanwise length) $\times 0.37 \mathrm{~m}$ (height) $\times 1.11 \mathrm{~m}$ (streamwise length) with corresponding grid points chosen as $512 \times 512 \times 1536$. The streamwise length is set as 48 mouth diameters such that it is sufficient to capture the droplet condensation in the puff and the Lagrangian statistics of the droplets. In a real cough, emissions can indeed reach further as shown in the work by Ref. [26]. However, at long distances many other issues play a role, such as ventilation, the presence of other people, etc. Moreover, covering even larger volumes will make our numerical simulations even more expensive. This, together with the many extra flow parameters we would have to consider, prevents us from doing so. Finally, obviously we cannot answer any questions about the infectivity of virus-laden droplets, viral loads at long distances, all of which are out of the scope of fluid mechanics. We model the mouth as a circular inlet centered at midheight of the domain and the cough temporal profile we apply is a gamma distribution as $\tilde{U}_{\text {cough }}(t)=U_{\text {cough }} \alpha t \exp (-\alpha t / 4)$, where $\alpha=60.9 \mathrm{~s}^{-1}$, such that the entire cough process lasts for about $0.6 \mathrm{~s}$. Based on the experimental measurement of Ref. [13], we seeded the respiratory event with droplets with initial diameters ranging between $10 \mu \mathrm{m}$ and roughly $1000 \mu \mathrm{m}$. We employ a similar droplet size distribution as Refs. [13,26] with 5000 droplets (see Fig. 1). The droplets are randomly positioned at the inlet area and evenly injected in time with the velocity matching the local inlet velocity. Droplets are assumed not to collide or coalesce since the estimates of the droplet volume fractions give $O\left(10^{-6}\right)$. Because the volume fraction considered is dilute, we chose not to couple the droplets to the momentum field, as such a coupling would have a negligible effect. Given that the droplet distribution is dilute and, as we will also show later, that the droplet condensation depends on the supersatured puff, we can infer that our results are insensitive to the exact droplets' spatial density and to the exact probability distribution of their sizes. These droplets also do not set the relative humidity of the puff, which at inflow is assumed to be $100 \%$, corresponding to saturated conditions from the lungs [44]. A detailed list of cough parameters and material properties of the gas and liquid phases is shown in Table I.

To interpolate gas quantities to the droplet locations, we employ the tricubic Hermitian interpolation scheme, which is sufficiently accurate for turbulent flows and comparable to $B$-spline interpolations $[45,46]$. The backwards forcing of the droplets onto the gas phase uses the trilinear projection onto the eight nearest nodes to the droplet location [47].

\section{EFFECT OF AMBIENT TEMPERATURE ON TURBULENT VAPOR PUFF AND DROPLET COUNTS}

First, we highlight the differences in the local RH values for two different $\theta_{\text {amb }}\left(10\right.$ and $\left.30^{\circ} \mathrm{C}\right)$ at $\mathrm{RH}_{\mathrm{amb}}=90 \%$. Note that $\theta_{\mathrm{amb}}$ and $\mathrm{RH}_{\mathrm{amb}}$ both constitute the control parameters of our study. 


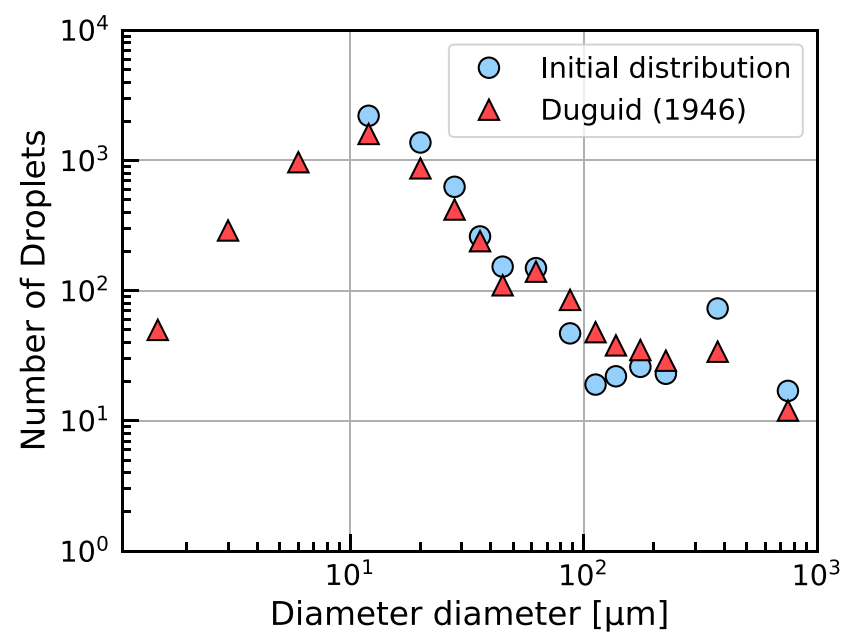

FIG. 1. Comparison of our employed initial droplet size distribution to that from Ref. [13]. Here, 5000 droplets are included in total. Note that a one-to-one comparison is not possible because the experimental measurements are taken after some time after expulsion, whereas our droplet distributions are prescribed as initial conditions for our DNS.

The local RH field is shown in Fig. 2 together with the instantaneous droplet distributions and instantaneous count histogram. At $\theta_{\mathrm{amb}}=30^{\circ} \mathrm{C}$, the local $\mathrm{RH}$ field is almost indistinguishable from $\mathrm{RH}_{\mathrm{amb}}$, as shown in Fig. 2(a). In contrast, at $\theta_{\mathrm{amb}}=10^{\circ} \mathrm{C}$ [Fig. 2(b)], the local $\mathrm{RH}$ field reaches supersaturation values larger than $100 \%$ (whitish color shading). In the count histograms in Fig. 2(b), most of the droplet diameters remain at least around $20 \mu \mathrm{m}$ throughout the spread distance,

TABLE I. List of cough parameters and the material properties of the surrounding gas and the droplet liquid. We in particular give the values of these parameters and material properties which we employed in the numerical simulations of this study. Droplets are assumed to contain pure water. The selected values are representative of a cough from experiments $[26,42,43]$.

\begin{tabular}{lll}
\hline \hline Cough properties & Symbols & \multicolumn{1}{c}{ Values } \\
\hline Temperature of vapor puff & $\theta_{\text {cough }}$ & $34{ }^{\circ} \mathrm{C}$ \\
Mean velocity of cough & $U_{\text {cough }}$ & $11.2 \mathrm{~m} / \mathrm{s}$ \\
Diameter of mouth & $D_{\text {mouth }}$ & $2.3 \mathrm{~cm}$ \\
Properties for gas phase & & \\
Diffusivity of water vapor in the gas phase & $D_{\text {vap }}$ & $2.5 \times 10^{-5} \mathrm{~m}^{2} / \mathrm{s}$ \\
Thermal diffusivity of gas & $D_{g}$ & $2.0 \times 10^{-5} \mathrm{~m}^{2} / \mathrm{s} \mathrm{for} \mathrm{air} \mathrm{at} 25^{\circ} \mathrm{C}$ \\
Air kinematic viscosity & $v_{\text {air }}$ & $1.562 \times 10^{-5} \mathrm{~m}^{2} / \mathrm{s}$ \\
Density of the gas & $\rho_{g}$ & $1.204 \mathrm{~kg} / \mathrm{m}^{3}$ \\
Thermal expansion coefficient & $\beta_{\theta}$ & $3.5 \times 10^{-3} / \mathrm{K}$ \\
Expansion coefficient for vapor mass fraction & $\beta_{c}$ & $0.5 \mathrm{~m} / \mathrm{kg} \times 1.204 \mathrm{~kg} / \mathrm{m}^{3} \approx 0.6$ \\
Specific heat capacity of gas & $c_{p, g}$ & $1 \mathrm{~kJ} /(\mathrm{kg} \cdot \mathrm{K})$ \\
Specific gas constant of water vapor & $R$ & $461.5 \mathrm{~J} /(\mathrm{kg} \cdot \mathrm{K})$ \\
Properties for droplets & & \\
Droplet density & $\rho_{l}$ & $993 \mathrm{~kg} / \mathrm{m}^{3}$ \\
Particle density parameter & $\beta$ & -0.9982 \\
Specific heat capacity of droplet & $c_{p, l}$ & $4.186 \mathrm{~kJ} /(\mathrm{kg} \cdot \mathrm{K})$ \\
Latent heat of water & $L$ & $2256 \mathrm{~kJ} / \mathrm{kg}$ \\
\hline \hline
\end{tabular}




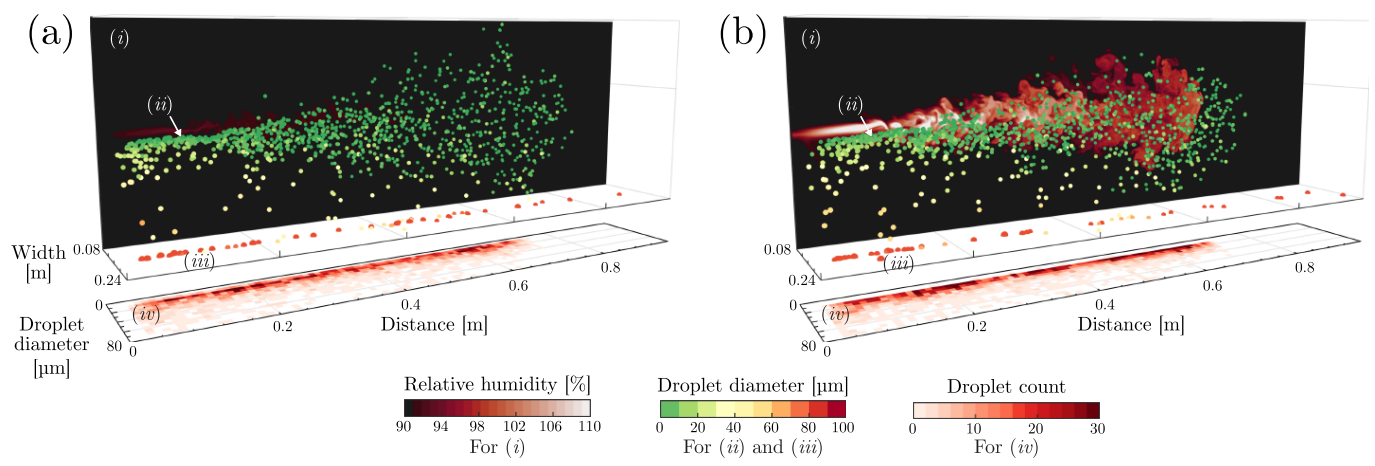

FIG. 2. Flow visualization snapshots from our direct numerical simulations of water droplets in a warm humid puff in ambient air at (a) $\theta_{\mathrm{amb}}=30^{\circ} \mathrm{C}$ and (b) $\theta_{\mathrm{amb}}=10^{\circ} \mathrm{C}$, both at $\mathrm{RH}_{\mathrm{amb}}=90 \%$. Corresponding movies can be seen in Movies S1 and S2 [48]. The snapshots show (i) vertical 2D planes of the local RH fields, (ii) the instantaneous droplets spatial distribution, (iii) the heavy large droplets which already fell on the ground, and (iv) the instantaneous droplet size histograms vs distance. The local RH planes are taken from the vertical midplane of the puff and are plotted on the background for clarity. Droplets are color coded by their instantaneous sizes. Initial droplet sizes are prescribed with a distribution similar to Ref. [13] (plotted in Fig. 1) and are injected evenly in time with the same local inflow velocity. The initial temperature of the droplets and puff is $34^{\circ} \mathrm{C}$. Both snapshots are taken at $0.6 \mathrm{~s}$ corresponding to the cutoff time of the puff. In the colder conditions of (b), the expelled humid puff oversaturates (seen as the lighter colored RH field and visible in Movie S2), which in turn dictates the growth of smaller droplets caught within the puff. Correspondingly, the droplet counts are confined within a narrower range of sizes in (b) as compared to (a) [see Fig. 3(a)].

whereas in Fig. 2(a), droplet diameters can go below $20 \mu \mathrm{m}$. This means that droplets expelled into colder ambient surroundings shrink slower because of the supersaturated local RH field. On the other hand, droplets expelled into hotter ambient surroundings shrink much more rapidly because the local RH rapidly undersaturates.

To illustrate the temperature sensitivity of the droplet size distributions, in Fig. 3, the distributions across three $\theta_{\mathrm{amb}}, 10,20$, and $30^{\circ} \mathrm{C}$ are shown at $t=0.6 \mathrm{~s}$ [Fig. 3(a)] and $1.2 \mathrm{~s} \mathrm{[Fig.} \mathrm{3(b)].} \mathrm{In} \mathrm{the}$ figure, the initial droplet size distribution (solid line) is also included for comparison. In Fig. 3(a), which corresponds to the end, or cutoff time, of the simulated puff, many smaller droplets $\lesssim 15 \mu \mathrm{m}$ already exist at $\theta_{\mathrm{amb}}=20$ and $30^{\circ} \mathrm{C}$ as compared to $\theta_{\mathrm{amb}}=10^{\circ} \mathrm{C}$. Interestingly, the histogram distribution of droplet diameters for $\theta_{\mathrm{amb}}=10^{\circ} \mathrm{C}$ is slightly higher and shifted to the right from the initial droplet size histogram, reflecting that a portion of the smaller droplets in fact grew. The reason for this increase is that due to the cold surrounding air, which can take up less vapor than the warm one, the puff gets supersaturated. This effect will be quantified in detail in Sec. V. Note that in Fig. 3, only the instantaneous droplet distribution (a subset of the 5000 droplets) is shown, corresponding to the associated time instant. The total number of droplets over the entire simulation is constant. At the later time of $t=1.2 \mathrm{~s}$ [Fig. 3(b)], most of the droplet sizes for $\theta_{\mathrm{amb}}=20$ and $30^{\circ} \mathrm{C}$ are shifted to the left towards much smaller sizes. Remarkably, the size distribution for $\theta_{\mathrm{amb}}=10^{\circ} \mathrm{C}$ remains roughly similar to the initially prescribed size distribution, with some lower counts at sizes $\lesssim 15 \mu \mathrm{m}$.

After having shown the distinct droplet shrinkage behavior under different ambient conditions, in the following section, we will further and in more detail quantify how the local fluid properties affect the shrinkage rate of the droplets.

\section{LAGRANGIAN STATISTICS OF THE DROPLETS}

For the following Lagrangian statistics, we focus on the droplets with an initial diameter around $15 \mu \mathrm{m}$, given that for those we observe the most pronounced temperature effect [see the count 

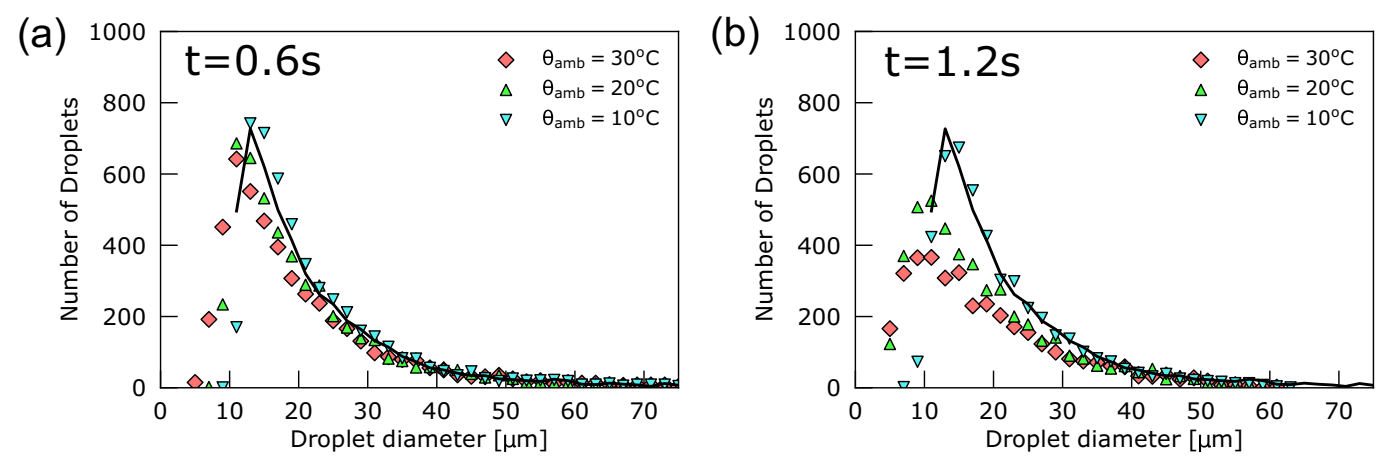

FIG. 3. Histogram of droplet sizes at (a) $t=0.6 \mathrm{~s}$ and (b) $1.2 \mathrm{~s}$ for $\theta_{\mathrm{amb}}=10,20,30^{\circ} \mathrm{C}$. As a point of reference, the solid lines denote the initial droplet size distributions based on the experimental measurement by Ref. [13] (see Appendix). The bin size of the histogram is $2 \mu \mathrm{m}$. At $t=0.6 \mathrm{~s}$ (a), the peak of the histogram for $\theta_{\text {amb }}=30^{\circ} \mathrm{C}$ marginally shifts down, whereas the overall histogram for $\theta_{\text {amb }}=10^{\circ} \mathrm{C}$ remains roughly similar to the initial distribution. The gap is more pronounced at $t=1.2 \mathrm{~s} \mathrm{(b):} \mathrm{For} \theta_{\mathrm{amb}}=30^{\circ} \mathrm{C}$, the peak of the histogram is halved from the initial peak, whereas in contrast, for $\theta_{\mathrm{amb}}=10^{\circ} \mathrm{C}$, the histogram still remains roughly unchanged. The relative invariance of the histogram for $\theta_{\mathrm{amb}}=10^{\circ} \mathrm{C}$ is due to the droplet growth dictated by the supersaturated vapor puff, which is in turn determined by cold and humid ambient conditions, as described in the main text.

histogram in Fig. 3(b)]. First, we track the droplet surface area $\propto d^{2}$ versus time as shown in Figs. 4(a) and 4(b). Note that droplets are expelled from the mouth at different time instants until the respiratory event stops $(0.6 \mathrm{~s}$ for the coughing event considered here). In order to compare the droplet statistics, we have shifted the time frame by their respective ejecting times.

For $\theta_{\mathrm{amb}}=30^{\circ} \mathrm{C}$ [Fig. 4(a)], the normalized surface area of the droplets $d^{2} / d_{\text {init }}^{2}$ decreases monotonically with time (the ensemble average of the value is depicted by the solid curve), which indicates pure evaporation of the expelled droplets. The surface area decreases linearly with time with the averaged droplet surface area being halved after $1 \mathrm{~s}$. Although a linear decline of the surface area is consistent with the classical $d^{2}$ law [24], one should note that the magnitude of the droplet shrinkage rate is still much smaller than that predicted by Wells [22]. Such a discrepancy is caused by the fact that the turbulent vapor puff engulfs small droplets and the puff contains higher RH than the ambient fluid [3,27].

Surprisingly, for the low $\theta_{\mathrm{amb}}$ of $10^{\circ} \mathrm{C}$ [Fig. 4(b)], the respiratory droplets evolve in a very different and distinct way: Instead of the pure evaporation during the time evolution of the droplets, there exists an initial stage (from the expelled time to about $0.4 \mathrm{~s}$ ) during which the droplets can grow with the averaged $d^{2}$ increasing by $10 \%$. This nonmonotonic trend of the shrinkage markedly differs from the classical $d^{2}$ law. At longer times, an effective $d^{2}$ decay will still prevail, though with a much slower decay (about 17 times slower here) than suggested by the Langmuir [24] $d^{2}$ decay. The departure from the $d^{2}$ decay is also clearly seen in Fig. 8 where we show the size evolution of $15-\mu \mathrm{m}$ droplets at various combinations of ambient temperatures and RH. One can presumably parametrize these departures, for example by application of mixing concepts on the humid puff as has been worked out for dense sprays [49-51]. It also clearly contradicts the well-accepted view that the respiratory droplets should simply evaporate, such as in Refs. [22,28,52,53], after expulsion. While the time over which the growth of $10 \%$ occurs only for approximately $0.3 \mathrm{~s}$, this time duration is half of the imposed puff duration. Therefore, it is argued that a majority of the droplets that are caught within the puff will in fact grow (at this $\theta_{\mathrm{amb}}$ and $\mathrm{RH}$ value) for a substantial duration of puff. Such a phenomenon of droplet condensation during the respiratory event is quantified owing to the Lagrangian statistics obtained from DNS, which has been neglected in the hitherto studies of droplet transmission exhaled into ambient surroundings. The focus of existing literature on droplet 
$\theta_{\text {amb }}=30^{\circ} \mathrm{C}$

(a)

(c)
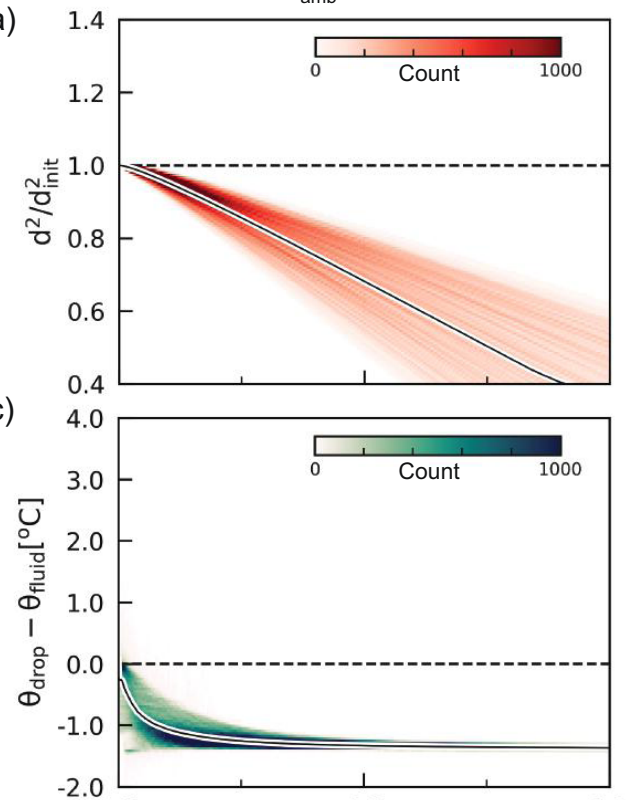

(e)

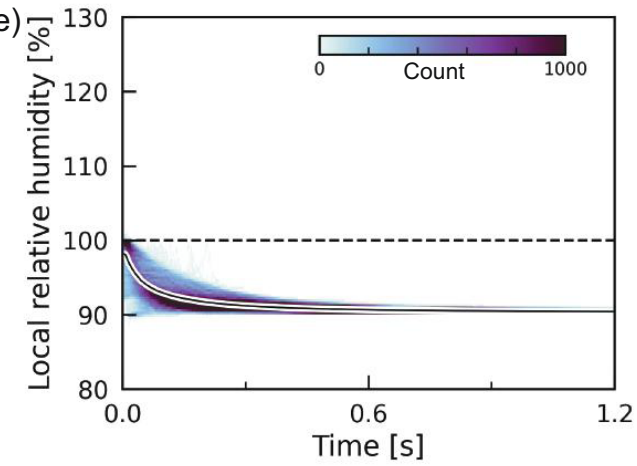

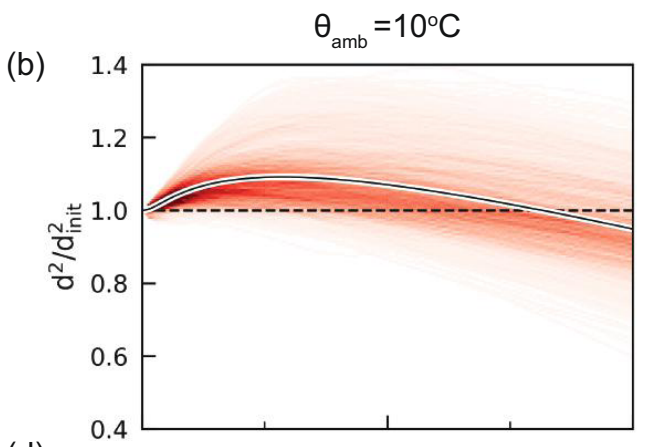

(d)
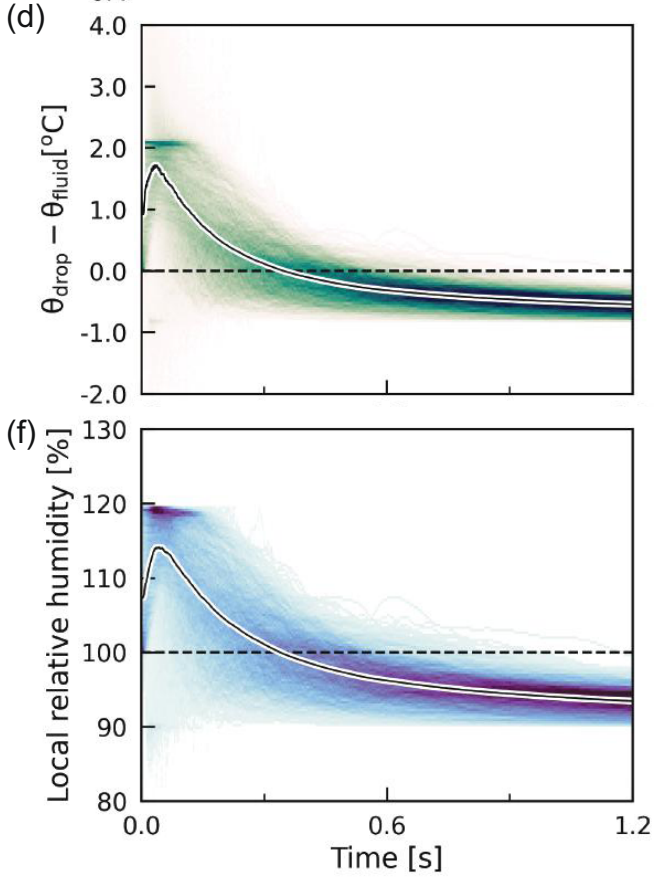

FIG. 4. Lagrangian statistics of droplets with initial droplet diameters $d_{\text {init }} \approx 15 \mu \mathrm{m}$ for $\theta_{\text {amb }}=30$ and $10{ }^{\circ} \mathrm{C}$. (a), (b) Change in droplet surface area $d^{2} / d_{\text {init }}^{2}$ vs time. (c), (d) Temperature difference between droplets and surrounding fluid, $\theta_{\text {drop }}-\theta_{\text {fluid }}$, vs time. (e), (f) Local RH vs time experienced by droplets. The time is shifted to the initial expelled time of each droplet. The ensemble average value is shown by the solid curve. The droplet counts are indicated by the colors (see inset color bars). For $\theta_{\mathrm{amb}}=30^{\circ} \mathrm{C}$ (a), droplets undergo pure evaporation after being expelled. Conversely, for $\theta_{\mathrm{amb}}=10^{\circ} \mathrm{C}(\mathrm{b})$, most droplets undergo growth in the initial stages and then evaporate. This growth in (b) is dictated by the supersaturated local RH values [see (f) and visible in Movie S2], which exceed 100\%.

condensation is typically in different setups, such as a pattern or dew formation on cold surfaces $[54,55]$, or employed as a noninvasive medical procedure for the retrieval of biomarkers from the lungs [56].

Another indicator that for $10^{\circ} \mathrm{C}$ the droplet size first increases is that the droplet temperature $\theta_{\text {drop }}$ increases relative to the fluid temperature at the location of the droplet $\theta_{\text {fluid }}$ [Fig. 4(d)]. As latent heat is released during droplet condensation for $10^{\circ} \mathrm{C}$ [Fig. 4(d)], the difference $\theta_{\text {drop }}-\theta_{\text {fluid }}$ increases during the early time. In contrast, $\theta_{\text {drop }}-\theta_{\text {fluid }}$ remains less than zero for $\theta_{\text {amb }}=30^{\circ} \mathrm{C}$ [Fig. 4 (c)], due to the latent heat needed for evaporation. Eventually, the droplet temperature saturates at a value lower than the fluid temperature. This is reasonable because the Jakob number, which is the ratio of 
the sensible heat to the latent heat due to the phase change, is much smaller than one $(0.04$ and 0.007 for $\theta_{\mathrm{amb}}=10$ and $30^{\circ} \mathrm{C}$, respectively). Therefore, the evaporation cooling effect plays a significant role here.

To understand why the droplets can grow during the early time, a key quantity to examine is the local RH experienced by the droplet. In Figs. 4(e) and 4(f), we compare the time evolution of the local RH at the droplet location for the two different $\theta_{\mathrm{amb}}$, showing quite different behaviors: For $30^{\circ} \mathrm{C}$, the local $\mathrm{RH}$ decreases monotonically to $\mathrm{RH}_{\mathrm{amb}}$ which is $90 \%$ in this case. However, for $10{ }^{\circ} \mathrm{C}$, the droplets first experience supersaturation within $0.4 \mathrm{~s}$ after expelling and the maximum $\mathrm{RH}$ reaches almost $115 \%$. This duration of having supersaturation is consistent with the duration of droplet growth, which reflects that it is the local supersaturation of the surrounding air which makes the droplets grow.

In fact, our daily life experience gives us intuition about this intriguing droplet condensation phenomenon. In cold and humid weather, one may observe the "white mist" coming out from the mouth while breathing or speaking. The physical explanation is that warm air can contain more moisture than cold air. Therefore, the exhaled warm vapor puff becomes supersaturated when it enters the cold ambient fluid, and the existing droplets and dust particles in air favor vapor condensation, both acting as nucleation sites. In our simulations, we only take the former into consideration and ignore the role of possible air impurities such as dust particles, in order to have well-defined conditions. Indeed, the importance of nucleation at the early stage of emission is very much unexplored. Studies on this effect are necessary and may give insight on the role of air quality to the dispersion of droplets. Such supersaturated conditions become more prominent if the temperature difference between the exhaled vapor and ambient fluid is larger. Here, we have clearly demonstrated the significance of supersaturation in the respiratory event that leads to the possibility of vastly different droplet dynamics.

\section{THEORETICAL MODEL PREDICTING RELATIVE HUMIDITY PROFILES AND SUPERSATURATION CRITERIA}

Motivated by the strong effect of the local RH field on the droplets, here we propose a simple model to calculate the local RH. Several assumptions first need to be made to justify this model. First, the axial scalar quantities (vapor mass fraction and temperature) are the dominant fluid properties that determine droplet growth/evaporation, and second, the exhaled puff at the time of $t=0.2 \mathrm{~s}$ exhibits jetlike properties such that the quasistationary mean properties of the puff admit self-similarity. Here, we employ the Antoine equation in order to compute the saturated vapor mass fraction for a given temperature field [57]. In short, given the local values of vapor mass fraction and temperature along the jet centerline, the axial RH can be directly computed.

Following the theory for axisymmetric turbulent jets [58-60], the axial scalar concentration distribution (say $\Phi_{\text {axial }}$ ) can be approximated by the relation

$$
\frac{\Phi_{\text {axial }}}{\Phi_{\text {inlet }}}=\frac{5}{1+x / d_{\text {inlet }}},
$$

where $\Phi_{\text {inlet }}$ is the inlet scalar concentration value, and $x / d_{\text {inlet }}$ is the axial distance normalized by the inlet diameter. Since this model diverges as $x / d_{\text {inlet }} \rightarrow 0$, we further impose the requirement that $\max \left[\Phi_{\text {axial }} / \Phi_{\text {inlet }}\right]=1$. Both the exhaled vapor mass fraction and temperature are modeled using Eq. (9) along the axis normal to the inlet.

To assess the validity of our assumptions, we plot the resulting model of the axial RH profiles at $t=0.2 \mathrm{~s}$ for different $\theta_{\mathrm{amb}}$ in Fig. 5. The agreement with our DNS (lighter curves) is quite promising, which is consistent with our assumption that the puff retains self-similar jetlike characteristics. This agreement lends some confidence to our ability to model the axial RH of our flow at a specific time instant, while the cough will approximate a turbulent puff or an interrupted jet at large $t$ after the injection velocity becomes negligible [61]. We note that the agreement at $t=0.2 \mathrm{~s}$ is also sensible since this corresponds to the peak in the puff signal which we numerically impose. Indeed, as the 


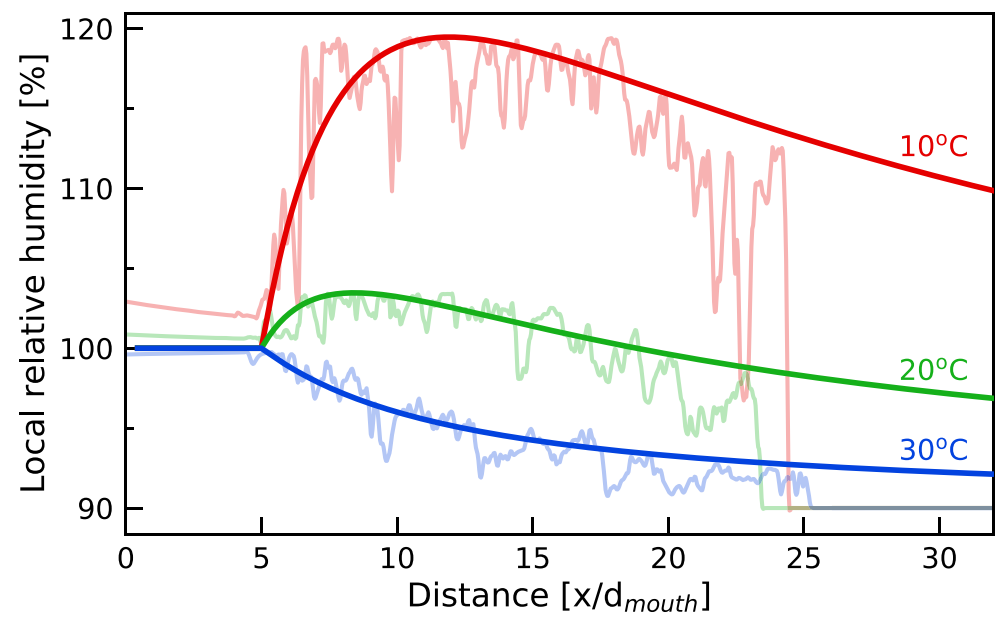

FIG. 5. Comparison of the results from our present DNS results (light curves) with those from the model for axial RH derived from Eq. (9) (smoother darker curves) at $t=0.2 \mathrm{~s}$ and $\mathrm{RH}_{\mathrm{amb}}=90 \%$. The data are plotted vs distance normalized by the mouth diameter, $x / d_{\text {mouth }}$. The different colored curves represent data for $\theta_{\text {amb }}$ of $10^{\circ} \mathrm{C}$ (red), $20^{\circ} \mathrm{C}$ (green), and $30^{\circ} \mathrm{C}$ (blue), respectively. The model RH curves all extend beyond $x / d_{\text {mouth }} \approx 25$ since the model assumes a fully developed jet, which is not the case for the present simulated case of a puff. However, the near-field axial model accurately captures the puff, implying quasistationary self-similar jet characteristics.
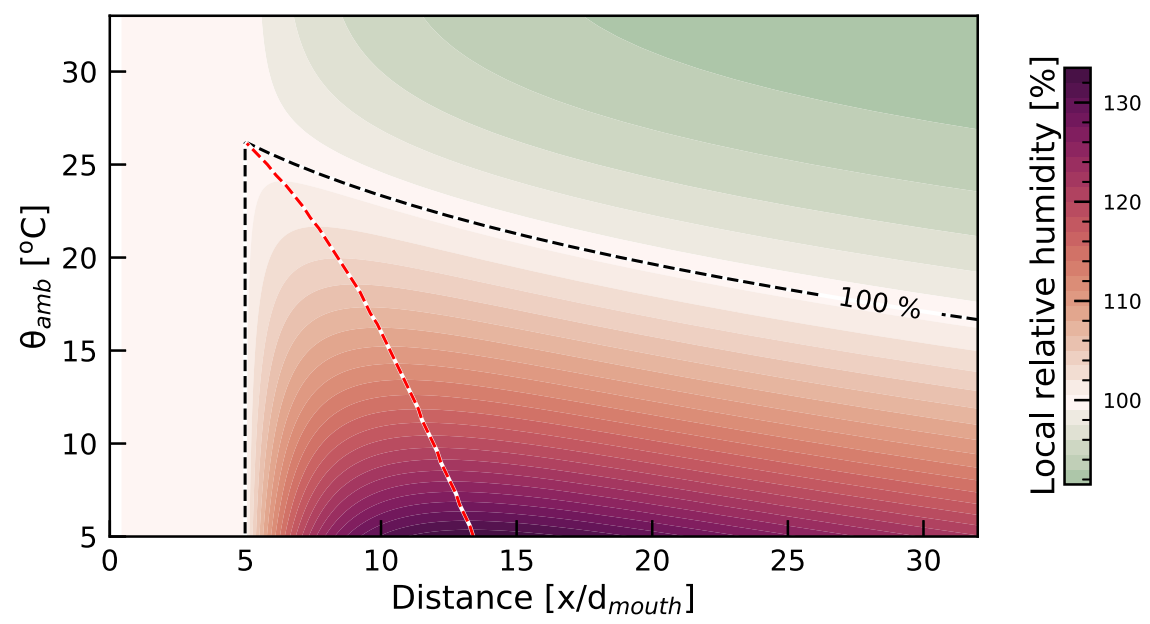

FIG. 6. $\theta_{\text {amb }}$ vs distance map for the RH model at $t=0.2 \mathrm{~s}$. The contours are computed for $\mathrm{RH}_{\mathrm{amb}}=90 \%$ with the color coded by the local RH. The boundary for RH oversaturation (local RH $>100 \%$ ) is shown by the dashed line. With decreasing $\theta_{\mathrm{amb}}$, the range with local $\mathrm{RH}>100 \%$ (width of the reddish area under the dashed curve) increases, which promotes growth of the smaller droplets because of the longer exposure to supersaturated regions. The most serious supersaturation conditions in this plot are for $\theta_{\text {amb }}=5^{\circ} \mathrm{C}$ and $x / d_{\text {mouth }} \approx 10-15$, namely beyond a local RH of $130 \%$. For a given $\theta_{\mathrm{amb}}$, the location of the maximum is marked by a dashed red line. 


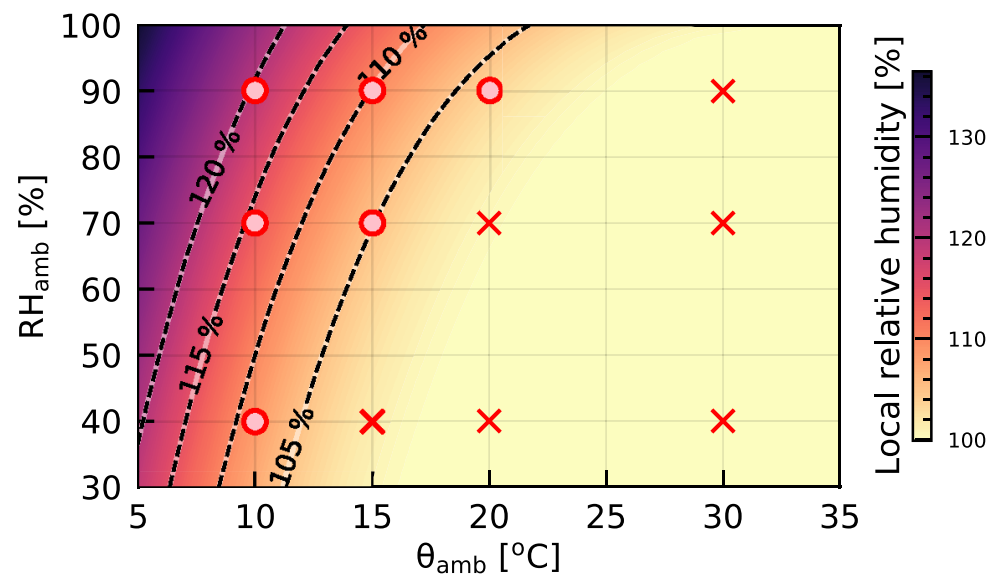

FIG. 7. Map of maximum local RH supersaturation values (see Fig. 6) for different $\theta_{\text {amb }}$ and $\mathrm{RH}_{\mathrm{amb}}$ based on the RH model. For the case of $R H_{\mathrm{amb}}=90 \%$ these are the values of the maximal local RH in Fig. 6 (red dashed curve). The values can be used as a gauge to indicate the tendency of droplets to experience supersaturation, given that the droplets are caught within the turbulent puff. The color is coded by the upper bounds for supersaturation. The plot indicates that in cool and humid environments, small droplets within a coughing puff will tend to grow once expelled. To assess the performance of our model, we also plot our DNS results for droplets with an initial size of $\approx 15 \mu \mathrm{m}$ and indicate growth or shrinkage using circle and cross symbols, respectively. (The detailed breakdown is plotted in Fig. 8.) A good agreement between our DNS and the RH model is seen.

imposed inlet velocity decays at $t>0.2 \mathrm{~s}$, the agreement will slightly degrade, but nevertheless, it does not influence our conclusion to employ Eq. (9) to compute the maximum local RH in the puff.

Based on the robustness of this model, we further propose the use of the maximum local RH as a metric to indicate the likelihood of the growth for small droplets. To illustrate this point, in Fig. 6, we plot the axial $\mathrm{RH}$ map for various $\theta_{\mathrm{amb}}$ versus distance at the fixed $\mathrm{RH}_{\mathrm{amb}}$ of $90 \%$. The dashed line shows the crossover between supersaturated and undersaturated axial RH values. One interesting observation from this model is that with decreasing $\theta_{\mathrm{amb}}$, the overall axial distance that experiences supersaturation increases. In other words, it is more likely that with decreasing $\theta_{\text {amb }}$ (at $\left.\mathrm{RH}_{\mathrm{amb}}=90 \%\right)$ small droplets will encounter longer supersaturated distances and grow in size. This likelihood to grow is important in understanding how far the droplets can be advected by the puff in the initial stages of the cough, before evaporating and dispersing.

Finally, we extend our idea of modeling the axial RH to map out the maximum local RH values for a range of $\mathrm{RH}_{\mathrm{amb}}$ and $\theta_{\mathrm{amb}}$. This map is shown in Fig. 7. From the figure, we find that the maximum local $\mathrm{RH}$ values are dependent on both $\mathrm{RH}_{\mathrm{amb}}$ and $\theta_{\mathrm{amb}}$. Several interesting observations can also be made from the figure. For instance, at given indoor ambient conditions of $\mathrm{RH}_{\mathrm{amb}}$ $=40 \%-50 \%$ (within acceptable occupancy comfort levels), the maximum local RH is at most $100 \%$ as long as $\theta_{\mathrm{amb}}>20^{\circ} \mathrm{C}$. However, once $\theta_{\mathrm{amb}}<20^{\circ} \mathrm{C}$, the local RH tends to supersaturate (RH $>100 \%)$, implying that in cooler environments, smaller droplets within the humid puff will first undergo supersaturation-driven growth in the early stages of the cough before evaporating upon fall-out. Such conditions become particularly exacerbated in outdoor environments, for example, in stadiums or sports fields during autumn or winter, when the temperatures tend to be colder. Similar conditions can also occur in crowded bus and train stations. Supersaturated conditions are more easily attainable (easily seen in exhaled mists) and therefore alter the respiratory droplet dynamics.

To substantiate our proposed model, in Fig. 7, we indicate on the plot 12 different control parameter combinations $\left(\mathrm{RH}_{\mathrm{amb}}\right.$ and $\left.\theta_{\mathrm{amb}}\right)$ for which droplets with an initial size of $\approx 15 \mu \mathrm{m}$ grow (indicated by a circle symbol) or shrink (indicated by a cross symbol) in our DNS and compare 

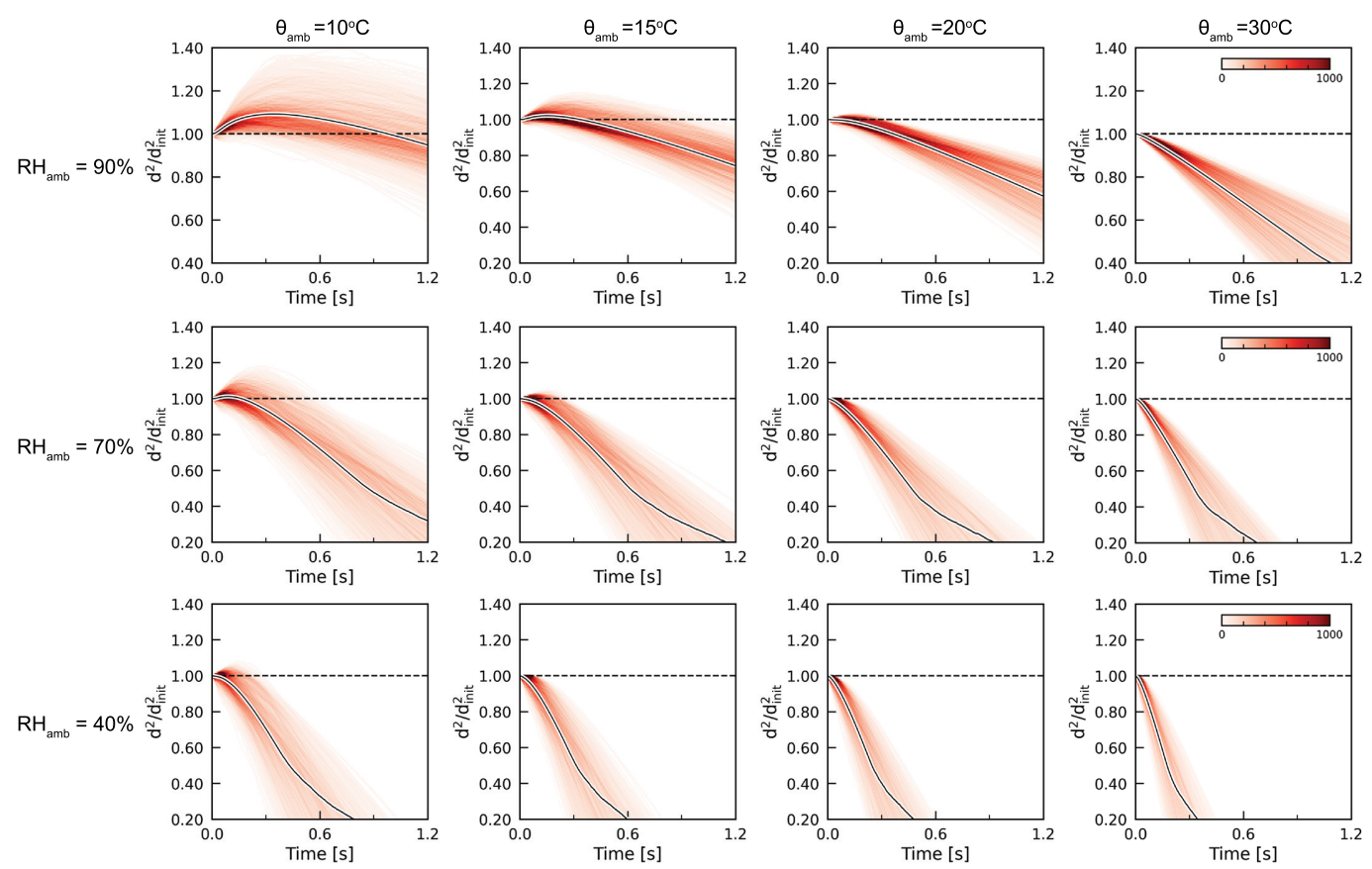

FIG. 8. Change of droplet surface area $d^{2} / d_{\text {init }}^{2}$ vs time for different $\mathrm{RH}_{\text {amb }}$ and $\theta_{\text {amb }}$ for initial droplet diameters $d_{\text {init }} \approx 15 \mu \mathrm{m}$. The ensemble average value is shown by the solid curve. For humid and cold ambient conditions, droplets tend to grow in the initial stages.

with the predictions of the model. (The profiles of $d^{2}$ versus time are provided in Fig. 8.) By comparing the symbols from the DNS and the map from the model, a clear correlation between growth and supersaturated local RH fields can be seen. Thus we propose that this map can be used as an indicator to determine the droplet size distributions of a similar cough.

It is also worth noting that we did not consider the influence of background wind, which is an important aspect both in ventilated indoor and outdoor environments. We also stress that we make no claims to correlate the growth or shrinkage of droplets with virus transmissions or infectivity. The subject of the viability of droplet sizes for viral media is still the topic of ongoing investigations in other communities and is not the focus of this work.

\section{CONCLUSIONS}

Through the Lagrangian statistics of the respiratory droplets, we have identified that when droplets are expelled from the mouth to the cold environment with high enough relative humidity, the droplet will first grow instead of immediately shrink, because the turbulent vapor puff becomes supersaturated. Crucially, we highlight the significance of this expelled turbulent puff on delaying the evaporation of respiratory droplets, and that the coherence of the puff is intimately dictated by both the ambient conditions and turbulent mixing. More importantly, we have provided a theoretical framework to accurately predict (i) how the relative humidity varies with distance in a respiratory puff, and (ii) the threshold of ambient temperature $\left(\theta_{\mathrm{amb}}\right)$ and relative humidity $\left(\mathrm{RH}_{\mathrm{amb}}\right)$ for which the supersaturated vapor field can result. Our theoretical model can also be applied to other respiratory events whenever there is jetlike transport, such as speaking [25], which is extremely relevant for asymptomatic and presymptomatic spreading of the coronavirus. In the situation of continuous speaking, when the hot vapor is continuously injected into the cold environment, we expect that 
the supersaturation zone may remain in front of the mouth for a long period of time. Other open questions remain, such as how much does the residence time of droplets of various sizes change over time and distance as a result of the evaporation/condensation processes. The result of the very distinct fate of respiratory droplets under different $\mathrm{RH}_{\mathrm{amb}}$ and $\theta_{\mathrm{amb}}$ may be instructive for developing timely strategies in mitigating the COVID-19 pandemic in different seasons. For example, during the winter time, where the maritime climates for many coastline European countries have a much higher $\mathrm{RH}_{\mathrm{amb}}$, the transmission in outdoor environments can presumably not be overlooked. However, we note that obviously also non-fluid-dynamical aspects affect the transmission and infectivity of viruses, such as from virology, epidemiology, and sociology, to name a few. These are not covered within our study and presumably interfere with our fluid dynamical results.

\section{ACKNOWLEDGMENTS}

The authors thank J. G. M. Kuerten for suggesting the idea to study droplet condensation. The authors also thank N. Hori for insightful discussions. This work was funded by the Netherlands Organisation for Health Research and Development (ZonMW), Project No. 10430012010022: "Measuring, understanding \& reducing respiratory droplet spreading," the ERC Advanced Grant DDD, No. 740479, and by several NWO grants. The funders have no role in study design, data collection, and analysis or decision to publish. The simulations were performed on the national e-infrastructure of SURFsara, a subsidiary of SURF cooperation, the collaborative ICT organization for Dutch education and research, the Irish Centre for High-End Computing (ICHEC), and Irene at Très Grand Centre de calcul du CEA (TGCC) under PRACE Project No. 2019215098.

[1] L. Bourouiba, The fluid dynamics of disease transmission, Annu. Rev. Fluid Mech. 53, 473 (2021).

[2] R. Mittal, R. Ni, and J. Seo, The flow physics of COVID-19, J. Fluid Mech. 894, F2 (2020).

[3] L. Bourouiba, Turbulent gas clouds and respiratory pathogen emissions: Potential implications for reducing transmission of COVID-19, J. Am. Med. Assoc. 323, 1837 (2020).

[4] S. Asadi, N. Bouvier, A. S. Wexler, and W. D. Ristenpart, The coronavirus pandemic and aerosols: Does COVID-19 transmit via expiratory particles? Aerosol Sci. Technol. 54, 635 (2020).

[5] L. Setti, F. Passarini, G. D. Gennaro, P. Barbieri, M. G. Perrone, M. Borelli, J. Palmisani, A. D. Gilio, P. Piscitelli, and A. Miani, Airborne transmission route of COVID-19: Why 2 meters/6 feet of inter-personal distance could not be enough, Int. J. Environ. Res. Public Health 17, 2932 (2020).

[6] P. Anfinrud, V. Stadnytskyi, C. E. Bax, and A. Bax, Visualizing speech-generated oral fluid droplets with laser light scattering, N. Engl. J. Med. 382, 2061 (2020).

[7] R. Zhang, Y. Li, A. L. Zhang, Y. Wang, and M. J. Molina, Identifying airborne transmission as the dominant route for the spread of COVID-19, Proc. Natl. Acad. Sci. USA 117, 14857 (2020).

[8] K. A. Prather, C. C. Wang, and R. T. Schooley, Reducing transmission of SARS-CoV-2, Science 368, $1422(2020)$.

[9] M. Jayaweera, H. Perera, B. Gunawardana, and J. Manatunge, Transmission of COVID-19 virus by droplets and aerosols: A critical review on the unresolved dichotomy, Environ. Res. 118, 109819 (2020).

[10] F. Yang, A. A. Pahlavan, S. Mendez, M. Abkarian, and H. A. Stone, Towards improved social distancing guidelines: Space and time dependence of virus transmission from speech-driven aerosol transport between two individuals, Phys. Rev. Fluids 5, 122501 (2020).

[11] R. K. Bhagat, M. S. Davies Wykes, S. B. Dalziel, and P. F. Linden, Effects of ventilation on the indoor spread of COVID-19, J. Fluid Mech. 903, F1 (2020).

[12] M. Abkarian and H. A. Stone, Stretching and break-up of saliva filaments during speech: A route for pathogen aerosolization and its potential mitigation, Phys. Rev. Fluids 5, 102301 (2020).

[13] J. P. Duguid, The size and the duration of air-carriage of respiratory droplets and droplet-nuclei, Epidemiol. Infect. 44, 471 (1946). 
[14] G. A. Somsen, C. van Rijn, S. Kooij, R. A. Bem, and D. Bonn, Small droplet aerosols in poorly ventilated spaces and SARS-CoV-2 transmission, Lancet Respir. Med. 8, 658 (2020).

[15] V. Stadnytskyi, C. E. Bax, A. Bax, and P. Anfinrud, The airborne lifetime of small speech droplets and their potential importance in SARS-CoV-2 transmission, Proc. Natl. Acad. Sci. USA 117, 11875 (2020).

[16] N. Moradian, H. D. Ochs, C. Sedikies, M. R. Hamblin, C. A. Camargo, J. A. Martinez, J. D. Biamonte, M. Abdollahi, P. J. Torres, J. J. Nieto et al., The urgent need for integrated science to fight COVID-19 pandemic and beyond, J. Transl. Med. 18, 1 (2020).

[17] H. C. Burridge, R. K. Bhagat, M. E. J. Stettler, P. Kumar, I. De Mel, P. Demis, A. Hart, Y. JohnsonLlambias, M.-F. King, O. Klymenko et al., The ventilation of buildings and other mitigating measures for COVID-19: a focus on wintertime, Proc. R. Soc. A 477, 20200855 (2021)..

[18] A. C. Lowen, S. Mubareka, J. Steel, and P. Palese, Influenza virus transmission is dependent on relative humidity and temperature, PLoS Pathog. 3, e151 (2007).

[19] J. Shaman and M. Kohn, Absolute humidity modulates influenza survival, transmission, and seasonality, Proc. Natl. Acad. Sci. USA 106, 3243 (2009).

[20] P. Mecenas, R. T. d. R. M. Bastos, A. C. R. Vallinoto, and D. Normando, Effects of temperature and humidity on the spread of COVID-19: A systematic review, PLoS ONE 15, e0238339 (2020).

[21] R. Wölfel, V. M. Corman, W. Guggemos, M. Seilmaier, S. Zange, M. A. Müller, D. Niemeyer, T. C. Jones, P. Vollmar, C. Rothe et al., Virological assessment of hospitalized patients with COVID-2019, Nature (London) 581, 465 (2020).

[22] W. F. Wells, On air-borne infection: Study II. Droplets and droplet nuclei, Am. J. Epidemiol. 20, 611 (1934).

[23] W. F. Wells and M. W. Wells, Air-borne infection, J. Am. Med. Assoc. 107, 1698 (1936).

[24] I. Langmuir, The evaporation of small spheres, Phys. Rev. 12, 368 (1918).

[25] M. Abkarian, S. Mendez, N. Xue, F. Yang, and H. A. Stone, Speech can produce jet-like transport relevant to asymptomatic spreading of virus, Proc. Natl. Acad. Sci. USA 117, 25237 (2020).

[26] L. Bourouiba, E. Dehandschoewercker, and J. W. M. Bush, Violent expiratory events: on coughing and sneezing, J. Fluid Mech. 745, 537 (2014).

[27] K. L. Chong, C. S. Ng, N. Hori, R. Yang, R. Verzicco, and D. Lohse, Extended Lifetime of Respiratory Droplets in a Turbulent Vapor Puff and Its Implications on Airborne Disease Transmission, Phys. Rev. Lett. 126, 034502 (2021).

[28] X. Xie, Y. Li, A. T. Y. Chwang, P. L. Ho, and W. H. Seto, How far droplets can move in indoor environments-revisiting the wells evaporation-falling curve, Indoor Air 17, 211 (2007).

[29] L. Liu, J. Wei, Y. Li, and A. Ooi, Evaporation and dispersion of respiratory droplets from coughing, Indoor Air 27, 179 (2017).

[30] B. Wang, H. Wu, and X.-F. Wan, Transport and fate of human expiratory droplets-A modeling approach, Phys. Fluids 32, 083307 (2020).

[31] S. Balachandar, S. Zaleski, A. Soldati, G. Ahmadi, and L. Bourouiba, Host-to-host airborne transmission as a multiphase flow problem for science-based social distance guidelines, Int. J. Multiphase Flow 132, 103439 (2020).

[32] R. Mittal, C. Meneveau, and W. Wu, A mathematical framework for estimating risk of airborne transmission of COVID-19 with application to face mask use and social distancing, Phys. Fluids 32, 101903 (2020).

[33] S. Chaudhuri, S. Basu, P. Kabi, V. R. Unni, and A. Saha, Modeling the role of respiratory droplets in COVID-19 type pandemics, Phys. Fluids 32, 063309 (2020).

[34] S. S. Diwan, S. Ravichandran, R. Govindarajan, and R. Narasimha, Understanding transmission dynamics of COVID-19-type infections by direct numerical simulations of cough/sneeze flows, Trans. Indian Natl. Acad. Eng. 5, 255 (2020).

[35] M. E. Rosti, M. Cavaoiloa, S. Olivieri, A. Seminara, and A. Mazzino, Turbulence role in the fate of virus-containing droplets in violent expiratory events, Phys. Rev. Research 3, 013091 (2021).

[36] J. Wang, F. D. Barba, and F. Picano, Direct numerical simulation of an evaporating turbulent diluted jet-spray at moderate Reynolds number, Int. J. Multiphase Flow 137, 103567 (2021). 
[37] R. Verzicco and P. Orlandi, A finite-difference scheme for three-dimensional incompressible flows in cylindrical coordinates, J. Comput. Phys. 123, 402 (1996).

[38] E. P. van der Poel, R. Ostilla-Mónico, J. Donners, and R. Verzicco, A pencil distributed finite difference code for strongly turbulent wall-bounded flows, Comput. Fluids 116, 10 (2015).

[39] M. R. Maxey and J. J. Riley, Equation of motion for a small rigid sphere in a nonuniform flow, Phys. Fluids 26, 883 (1983).

[40] A. Nguyen and H. J. Schulze, Colloidal Science of Flotation (CRC Press, Boca Raton, FL, 2003), Vol. 118.

[41] W. E. Ranz and W. R. Marshall, Jr., Evaporation from drops, Chem. Eng. Prog. 48, 173 (1952).

[42] J. K. Gupta, C.-H. Lin, and Q. Chen, Flow dynamics and characterization of a cough, Indoor Air 19, 517 (2009).

[43] J. W. Tang, T. J. Liebner, B. A. Craven, and G. S. Settles, A schlieren optical study of the human cough with and without wearing masks for aerosol infection control, J. R. Soc. Interface 6, S727 (2009).

[44] L. Morawska, G. Johnson, Z. Ristovski, M. Hargreaves, K. Mengersen, S. Corbett, C. Y. H. Chao, Y. Li, and D. Katoshevski, Size distribution and sites of origin of droplets expelled from the human respiratory tract during expiratory activities, J. Aerosol Sci. 40, 256 (2009).

[45] M. A. T. van Hinsberg, J. H. M. ten Thije Boonkkamp, F. Toschi, and H. J. H. Clercx, Optimal interpolation schemes for particle tracking in turbulence, Phys. Rev. E 87, 043307 (2013).

[46] R. Ostilla-Mónico, Y. Yang, E. P. van der Poel, D. Lohse, and R. Verzicco, A multiple-resolution strategy for direct numerical simulation of scalar turbulence, J. Comput. Phys. 301, 308 (2015).

[47] I. M. Mazzitelli, D. Lohse, and F. Toschi, On the relevance of the lift force in bubbly turbulence, J. Fluid Mech. 488, 283 (2003).

[48] See Supplemental Material at http://link.aps.org/supplemental/10.1103/PhysRevFluids.6.054303 for a three-dimensional visualization of the local relative humidity field, droplet spatial distribution, and droplet size distribution for $\theta_{\mathrm{amb}}=10$ and $30^{\circ} \mathrm{C}$.

[49] A. de Rivas and E. Villermaux, Dense spray evaporation as a mixing process, Phys. Rev. Fluids 1, 014201 (2016).

[50] E. Villermaux, A. Moutte, M. Amielh, and P. Meunier, Fine structure of the vapor field in evaporating dense sprays, Phys. Rev. Fluids 2, 074501 (2017).

[51] E. Villermaux, Mixing versus stirring, Annu. Rev. Fluid Mech. 51, 245 (2019).

[52] M. Nicas, W. W. Nazaroff, and A. Hubbard, Toward understanding the risk of secondary airborne infection: Emission of respirable pathogens, J. Occup. Environ. Hyg. 2, 143 (2005).

[53] L. C. Marr, J. W. Tang, J. Van Mullekom, and S. S. Lakdawala, Mechanistic insights into the effect of humidity on airborne influenza virus survival, transmission and incidence, J. R. Soc. Interface 16, 20180298 (2019).

[54] D. Beysens and C. M. Knobler, Growth of Breath Figures, Phys. Rev. Lett. 57, 1433 (1986).

[55] D. Beysens, The formation of dew, Atmos. Res. 39, 215 (1995).

[56] I. Horváth, J. Hunt, P. J. Barnes et al., Exhaled breath condensate: methodological recommendations and unresolved questions, Eur. Respir. J. 26, 523 (2005).

[57] E. Russo, J. G. Kuerten, C. van der Geld, and B. J. Geurts, Water droplet condensation and evaporation in turbulent channel flow, J. Fluid Mech. 749, 666 (2014).

[58] J. O. Hinze and B. G. Van Der Hegge Zijnen, Transfer of heat and matter in the turbulent mixing zone of an axially symmetrical jet, Flow, Turbul. Combust. 1, 435 (1949).

[59] H. Schlichting, Boundary-Layer Theory (Springer, Berlin, 1968), Vol. 960.

[60] Y. Antoine, F. Lemoine, and M. Lebouché, Turbulent transport of a passive scalar in a round jet discharging into a co-flowing stream, Eur. J. Mech. B Fluids 20, 275 (2001).

[61] J. Wei and Y. Li, Human cough as a two-stage jet and its role in particle transport, PLoS ONE 12, e0169235 (2017). 